\title{
Kasuistiken
}

Rechtsmedizin 2021 · 31:135-139 https://doi.org/10.1007/s00194-020-00448-3 Online publiziert: 3. Dezember 2020 (c) Der/die Autor(en) 2020

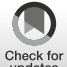

F. Zack ${ }^{1,8} \cdot$ P. Warnke ${ }^{2} \cdot$ J. Manhart ${ }^{1} \cdot$ A. Angeli ${ }^{3} \cdot$ H. Nizze ${ }^{4} \cdot$ J. O. Rudnick ${ }^{5} \cdot$

I. Steinhagen ${ }^{6} \cdot$ V. Kolbe ${ }^{1} \cdot$ M. Hammer ${ }^{7}$ A. Büttner ${ }^{1}$

'Institut für Rechtsmedizin, Universitätsmedizin Rostock, Rostock, Deutschland

${ }^{2}$ Institut für Medizinische Mikrobiologie, Virologie und Hygiene, Universitätsmedizin Rostock, Rostock, Deutschland

${ }^{3}$ Zoologischer Garten Rostock, Rostock, Deutschland

${ }^{4}$ Institut für Pathologie, Universitätsmedizin Rostock, Rostock, Deutschland

${ }^{5}$ Tierklinik Rostock, Rostock, Deutschland

${ }^{6}$ Institut für Diagnostische und Interventionelle Radiologie, Kinder- und Neuroradiologie,

Universitätsmedizin Rostock, Rostock, Deutschland

${ }^{7}$ Tierärztliche Praxis Bentwisch, Bentwisch, Deutschland

${ }^{8}$ Rostock, Deutschland

\section{Enterokolitis als Ursache eines akuten unerwarteten Todes eines Orang-Utans in Zoohaltung}

\section{Klärung der Todesursache durch Rechtsmediziner mit Unterstützung von Humanmedizinern weiterer Fachrichtungen}

unterstützt, z. B. in Abstammungsfragen bei Bonobos aus dem Kölner Zoo [3].

lich, dass Humanmediziner bei der Feststellung der Todesursache von Wildoder Zootieren die Veterinärmediziner unterstützen. Zudem kommen in der Rechtsmedizin gelegentlich Obduktionen von Tieren oder toxikologische Untersuchungen von Tierasservaten im Auftrag der Staatsanwaltschaft vor, z. B. bei Verstößen gegen das Tierschutzgesetz. An der Universitätsmedizin Rostock wurden insbesondere durch Rechtsmediziner bereits Wildtiere wie ein illegal abgeschossener Wolf, Seeadler mit Bleivergiftungen, ein Fischadler als Verursacher eines Kleinflugzeugabsturzes und mit Ethanol vergiftete Fische untersucht $[1,2]$. Weiterhin wurden hier vereinzelt auch Primaten aus dem heimischen Zoo wie z.B. ein weiblicher Gorilla mit chronischer Enteropathie und ein weiblicher Orang-Utan mit Cor pulmonale bei hochgradigen Bronchiektasien obduziert. Auch in anderen Instituten wurde die Veterinärmedizin von Wildund Zootieren durch Humanmediziner

\section{Fallbericht}

\section{Anamnese}

Das Orang-Utan-Weibchen Dinda wurde im Juli 2006 im Primatenrettungszentrum Wareham, Dorset (UK), geboren. In Taiwan war ihre Mutter von Behörden beim Versuch eines illegalen Tierhandels konfisziert, ihr Vater auf einer Straße gehend aufgegriffen worden. Nach der Geburt kümmerte sich die Mutter nicht um Dinda, sodass das Pflegepersonal den $1,97 \mathrm{~kg}$ schweren Orang-Utan-Säugling mit der Flasche aufzog. Seit ihrem ersten Lebensjahr litt Dinda an rezidivierenden Enteropathien. Dabei wurden als Erreger Entamöben, Escherichia coli und $\mathrm{Ba}$ lantidium nachgewiesen. In der Zeit zwischen 2006 und 2015 wurden mindestens 11 Durchfallerkrankungen bei Dinda aktenkundig dokumentiert.

Im Dezember 2015 zog Dinda auf Empfehlung des Europäischen Erhal- tungszuchtprogramm (EEP) aus dem Primatenzentrum Wareham, Dorset, in den Zoologischen Garten nach Rostock, Deutschland. Zwei Jahre später wurde Dinda mit 11 Jahren Mutter eines gesunden Orang-Utan-Weibchens. Von 2015 bis 2019 wurden bei Dinda keine ernsthaften gesundheitlichen Probleme festgestellt.

Am 03.02.2020 zeigte das Tier Inappetenz und einen reduzierten Bewegungsdrang. Beim Kotabsatz fanden sich schleimige Beimengungen. Da in den nächsten beiden Tagen keine Besserung eintrat, erfolgte am 05.02.2020 eine tierärztliche Untersuchung in intramuskulärer Injektionsnarkose. Das Orang-Utan-Weibchen hatte eine Körpertemperatur von $36,8^{\circ} \mathrm{C}$ und eine Herzfrequenz von $86 \mathrm{Schlägen/min.} \mathrm{In}$ der Sonographie zeigten sich sowohl auffällige Flüssigkeit im Zäkum als auch eine teilweise verbreiterte Kolonwand. Es wurde die Verdachtsdiagnose „chronische Kolitis“ geäußert. Nach der Narkose wachte Dinda ohne Probleme auf. Am Morgen des 06.02.2020 wurde Dinda 


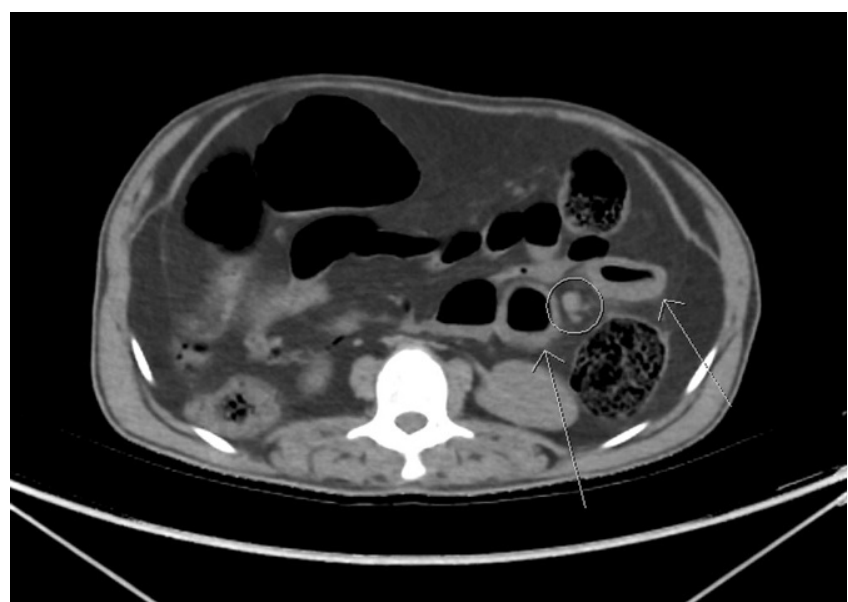

Abb. $1 \Delta$ Abdomen mit Verbreiterung der Kolonwand (Pfeile), Vergrößerung eines Lymphknotens (Kreis) und Dilatation des Zäkums (links oben) in der postmortalen Computertomographie

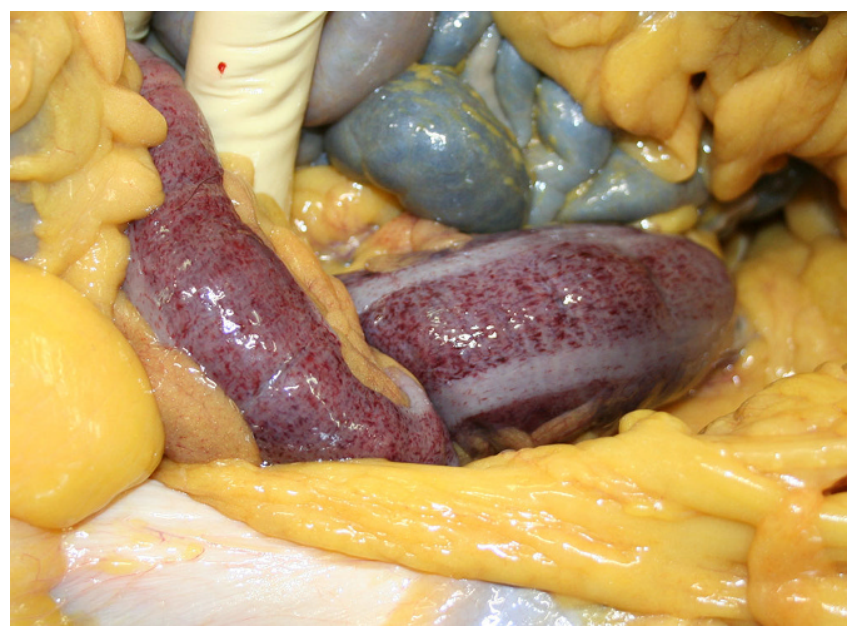

Abb. 3 ॥ Zahlreiche punktförmige Blutungen der Serosa des Kolons

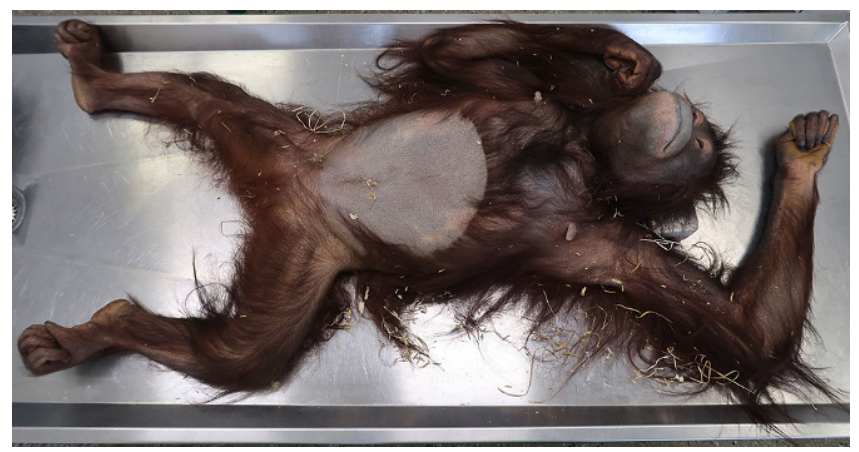

Abb. $2 \Delta$ Der weibliche Orang-Utan vor der Obduktion. Der rasierte Bauch aufgrund einer Sonographieuntersuchung vom Vortag

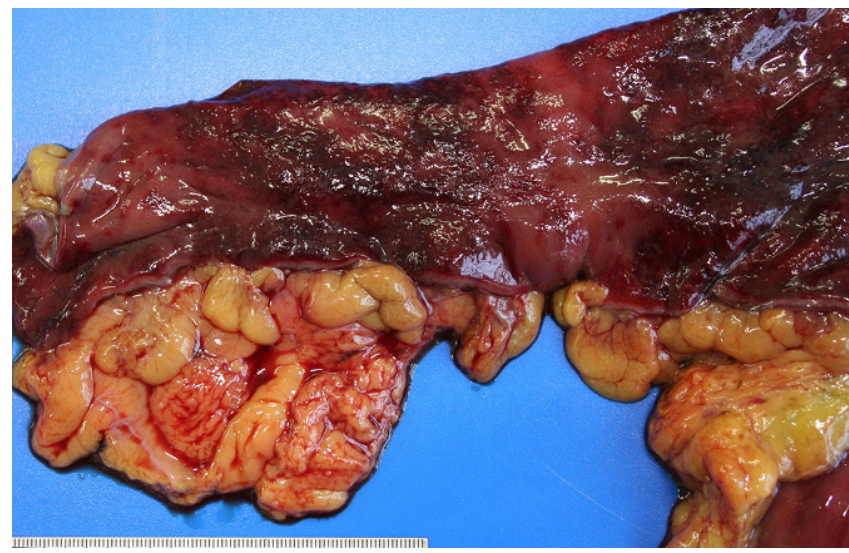

Abb. $4 \Delta$ Das Colon descendens mit Blutungen der Mukosa und Fibrose der Submukosa von einem Tierpfleger in ihrem Gehege tot aufgefunden. Alle anderen Affen des Rostocker Zoos, die mit Dinda im geräumigen „Darwineum“lebten, wiesen keine Krankheitssymptome auf. Nachfolgend wandte sich der Zoologische Garten, der mit der Universität Rostock kooperiert, mit der Bitte um eine schnellstmögliche Klärung der Todesursache an das Institut für Rechtsmedizin.

\section{Postmortale Computertomo- graphie und Obduktion}

Einen Tag nach dem Tod wurden an der Universitätsmedizin Rostock eine postmortale computertomographische Untersuchung (• Abb. 1) und eine Obduktion des Orang-Utans von Human- medizinern vorgenommen. Dinda war $123 \mathrm{~cm}$ lang und wog $46 \mathrm{~kg}$ (• Abb. 2). Die Untersuchungen zeigten eine chronische Kolitis mit regionalen Wandverdickungen und eine akute Kolitis mit massiver Blutung in das Darmlumen, bevorzugt im Zäkum und im Colon descendens (• Abb. 3, 4 und 5). Als Zeichen eines großen Blutverlustes wurden fleckförmige subendokardiale Blutungen des linken Ventrikels und blutarme parenchymatösen Organe festgestellt. Die übrigen Organe waren makroskopisch unauffällig.

\section{Histologische Untersuchungen}

Die histologischen Untersuchungen des Kolons zeigten eine hochgradige Fibro- se der Submukosa, ausgeprägte Schleimhautnekrosen, Hämorrhagien der Mukosa sowie eine geringgradige granulozytäre und lymphozytäre Infiltration der Submukosa (• Abb. 6, 7 und 8). Die histologischen Untersuchungen der anderen Organe zeigten keine auffälligen pathologischen Befunde.

\section{Mikrobiologische Untersuchungen}

Eine Gewebsprobe der Kolonwand wurde im Institut für medizinische Mikrobiologie, Virologie und Hygiene der Universitätsmedizin Rostock positiv auf DNA von enteroinvasiver Escherichia coli (EIEC), enteropathogener Escherichia coli (EPEC) sowie enteroaggregativer 
Escherichia coli (EAEC) getestet. Dabei soll auch erwähnt werden, dass die PCR herstellerseitig nicht für tierische oder postmortale Proben validiert war.

\section{Todesursache}

In der Gesamtbewertung der Ergebnisse der Obduktion sowie der histologischen und mikrobiologischen Untersuchungen wurde eine akute bakterielle Enterokolitis mit massiver Blutung bei vorbestehender chronischer Kolitis als Todesursache diagnostiziert.

\section{Diskussion}

Während bei freilebenden Orang-Utans auch nichtnatürliche Todesursachen wie Schussverletzungen, Vergiftungen oder Folgen schwerer Verletzungen vorkommen, wurden bei Orang-Utans in Zoohaltungen bevorzugt natürliche Todesursachen festgestellt $[4,5]$. Bei einer selektiven Literaturrecherche wurden keine Übersichtsarbeiten über Todesursachen von Orang-Utans gefunden. Hingegen gibt es besonders bei Zootieren zahlreiche Fallbeschreibungen, wie z.B. der Tod durch Coxsackie-B4-Myokarditis, Peritonitis nach Ruptur eines Divertikels, Pneumonie bei Streptococcusanginosus-Infektion, „airsacculitis“ und Pneumonie [6-10]. Auch Enteropathien bei Orang-Utans sind keine Seltenheit [11].

Aufgrund fehlender bzw. nicht umfassend belegter wissenschaftlicher Erkenntnisse in der Veterinärmedizin beziehen sich die nachfolgenden Diskussionspunkte auf das umfangreiche Fachwissen aus der Humanmedizin. Dabei ist beim Vergleich zwischen Mensch und Orang-Utan durchaus auch eine kritische Zurückhaltung geboten. Auf der anderen Seite zeigen die zitierten Todesursachen bei Orang-Utans in Zoohaltung, dass diese ausnahmslos auch in der Humanmedizin vorkommen.

Sowohl akute als auch chronische Enteropathien sind multifaktoriell bedingt. Aus der Vorgeschichte Dindas wurde bekannt, dass das nur 13 Jahre alt gewordene Tier seit dem ersten Lebensjahr an rezidivierenden Durchfallerkrankungen gelitten hatte. Das Geburtsgewicht für Orang-

Rechtsmedizin 2021 · 31:135-139 https://doi.org/10.1007/s00194-020-00448-3

(c) Der/die Autor(en) 2020

F. Zack · P. Warnke · J. Manhart · A. Angeli · H. Nizze · J. O. Rudnick · I. Steinhagen · V. Kolbe · M. Hammer · A. Büttner

Enterokolitis als Ursache eines akuten unerwarteten Todes eines Orang-Utans in Zoohaltung. Klärung der Todesursache durch Rechtsmediziner mit Unterstützung von Humanmedizinern weiterer Fachrichtungen

\section{Zusammenfassung}

Es wird von einem 13 Jahre alt gewordenen weiblichen Orang-Utan berichtet, der 4 Tage vor dem Tod an einer akuten Enterokolitis erkrankte. Die tierärztliche Ultraschalluntersuchung einen Tag vor dem Tod erbrachte den Nachweis von vermehrter Flüssigkeit im Zäkum und einer abschnittsweise verdickten Kolonwand. Nach dem für die Veterinärmediziner und die Zoomitarbeiter unerwarteten Todeseintritt erfolgte eine Obduktion durch Rechtsmediziner der Universitätsmedizin Rostock mit Unterstützung von Ärzten weiterer Fachrichtungen. Dabei wurde als Todesursache eine akute Escherichiacoli-Enterokolitis mit massiver Blutung in den Darm festgestellt. Begünstigend oder ursächlich für die letale Enterokolitis dürften wahrscheinlich zahlreiche rezidivierende Durchfallerkrankungen in der Anamnese des Orang-Utans als Folge eines Verweigerns des Stillens durch die Mutter nach der Geburt gewesen sein.

Schlüsselwörter

Menschenaffe - Darmblutung - Obduktion . Histologie · Escherichia coli

\section{Enterocolitis as the cause of sudden unexpected death in a captive orangutan (Pongo pygmaeus). Clarification of the cause of death by forensic medicine with the support of other medical specialists}

\section{Abstract}

This report reviews the death of a 13year-old female orangutan that died in captivity 4 days after suffering from acute enterocolitis. Increased fluid in the cecum and a thickened colon were detected in a veterinary ultrasound examination 1 day before the primate died. An autopsy was performed by forensic pathologists from the University of Rostock. It was determined that the orangutan died of an acute Escherichia coli enterocolitis with a massive intestinal hemorrhage. Recurrent diarrheal diseases caused by the orangutan's mother refusing to breastfeed may have promoted or caused the fatal enterocolitis.

\section{Keywords}

Great ape $\cdot$ Intestinal hemorrhage $\cdot$ Autopsy . Histology · Escherichia coli
Utans beträgt zwischen 1,5 und $2 \mathrm{~kg}$, sodass Dinda mit 1,97 kg ein Normgewicht aufgewiesen hatte. Hingegen spricht das Fehlen der Muttermilch durch das Verweigern des Stillens durch Dindas Mutter dafür, dass dies ein wesentlicher Risikofaktor für die Anfälligkeit des Tieres für Enteropathien gewesen war. Muttermilch ist die optimale Ernährung für Säuglinge und genau auf die Bedürfnisse der Neugeborenen abgestimmt. Sie ist nicht nur eine Nahrungsquelle, sondern enthält auch eine Vielzahl biologisch aktiver Bestandteile für die Entwicklung des Immunsystems und der Darmmikrobiota [12]. Säuglinge, die nicht mit Muttermilch ernährt werden, haben ein erhöhtes Risi- ko für Infektionserkrankungen. In der Humanmedizin ist bekannt, dass sowohl die Milch der leiblichen Mutter als auch gespendete Muttermilch eine Reduktion der Inzidenz der nekrotisierenden Enterokolitis der Säuglinge bewirkt. In Fällen einer nekrotisierenden Enterokolitis bei Säuglingen wird als Therapie die Gabe von Muttermilch empfohlen [13, 14].

Aber auch die letale Enterokolitis durch die festgestellten pathogenen Escherichia-coli(E.-coli)-Bakterien hatte mit einer hohen Wahrscheinlichkeit einen Bezug auf die fehlende Muttermilch nach der Geburt. Dinda hatte nachweislich zwischen 2006 und 2015 elf dokumentierte Enteropathien. 


Kasuistiken

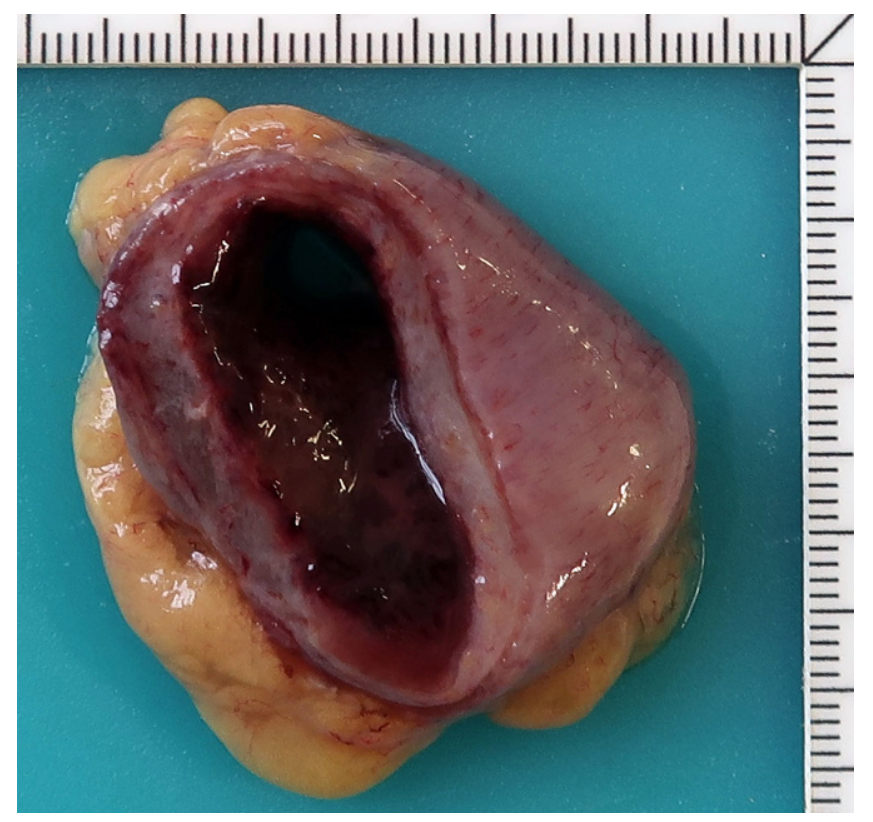

Abb. $5 \Delta$ Querschnitt des Colon descendens mit Fibrose der Submukosa und hämorrhagischer Mukosa

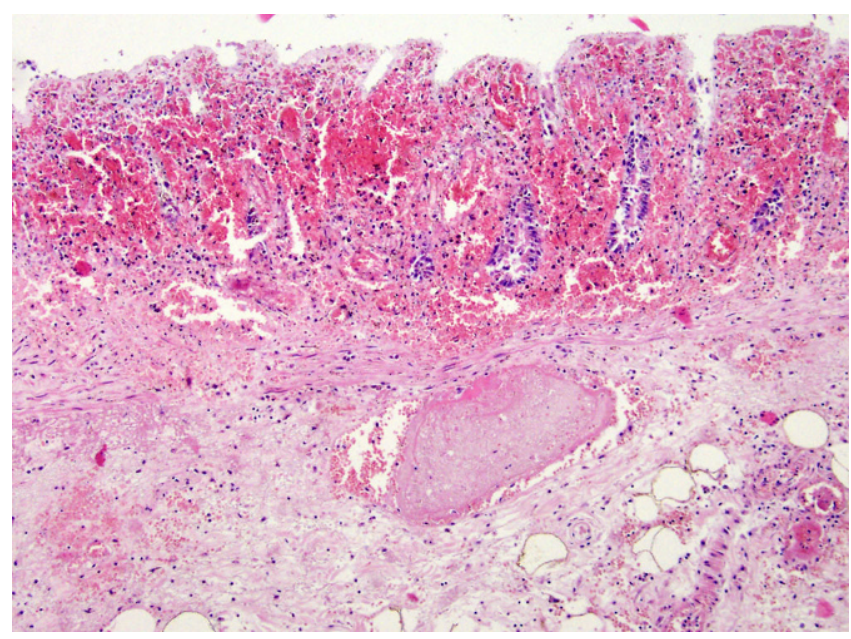

Abb. $7 \triangle$ Ausgedehnte Hämorrhagien der Mukosa und ein alter Mikrothrombus in der Submukosa. HE-Färbung, Vergr. 100:1

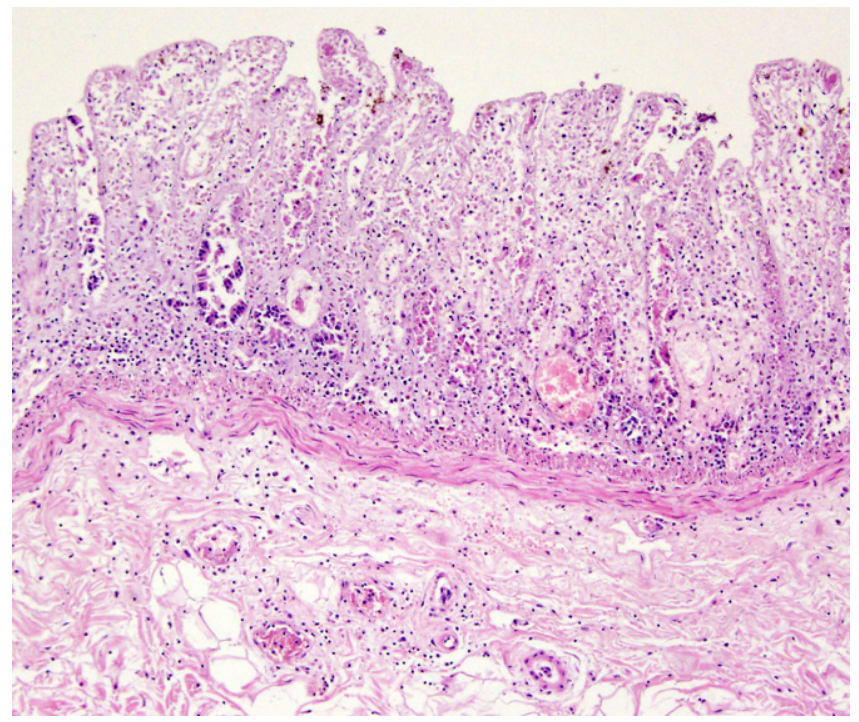

Abb. $6 \Delta$ Ausgedehnte Epithelnekrosen der Mukosa und geringgradige entzündliche Reaktion nahe der Lamina muscularis mucosae. HE-Färbung, Vergr. 100:1

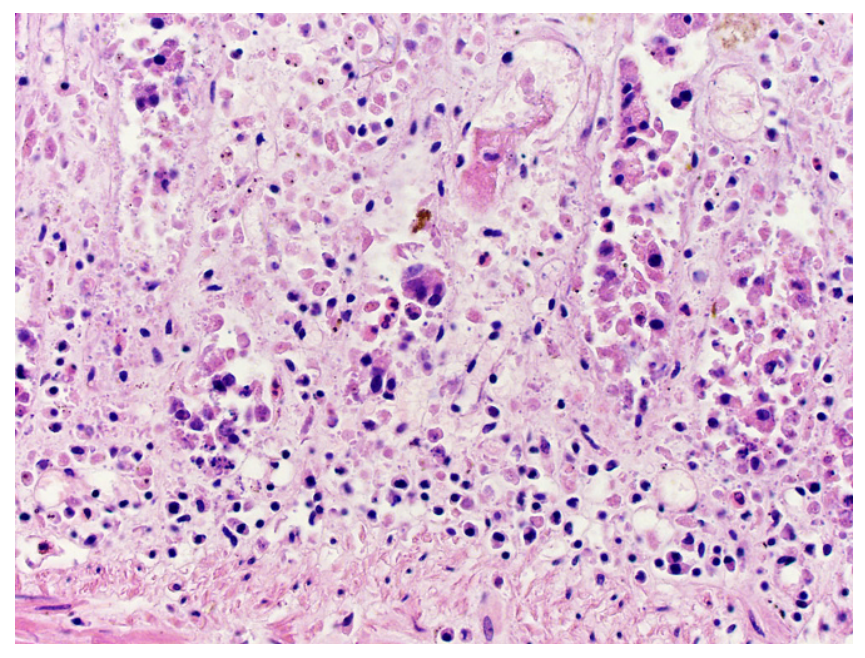

Abb. 8 ॥ Geringgradige Entzündungsinfiltrate bevorzugt aus Lymphozyten und Granulozyten in der Mukosa. HE-Färbung, Vergr. 400:1
Aus der Humanmedizin ist bekannt, dass rezidivierend auftretende Durchfallerkrankungen die Empfänglichkeit/ Anfälligkeit eines Patienten für pathogene E.-coli-Bakterien erhöhen [15]. Die makroskopisch und histologisch festgestellte Fibrose der Submukosa des Darmes ist am ehesten auf die zahlreichen Enteropathien zurückzuführen.

Die Ergebnisse der mikrobiologischen Untersuchungen stützen die Ergebnisse der Obduktion und der histologischen Untersuchungen [16]. Es bleibt unklar, ob der Nachweis der 3 Gene aus 3 ver- schiedenen E.-coli-Stämmen oder aus einem Stamm resultierte, der alle $3 \mathrm{Ge}$ ne enthielt. Ein Beispiel eines E.-coliStammes, der mehrere Virulenzprofile kombinierte, war die Ursache für einen schweren Ausbruch des hämolytisch-urämischen Syndroms und des hämorrhagischen Durchfalls 2011 in Europa und Nordamerika [17].

Dass Darmerkrankungen auch zu plötzlichen unerwarteten Todesfällen beim Menschen führen können, ist bekannt [18] Auffällig war im vorgestellten Fall die rasante Geschwindigkeit, mit der die Enterokolitis zum Tod führte. Den Grund dafür erbrachte die Obduktion. Die Toxine der pathogenen Bakterien führten zu ausgeprägten Nekrosen der Mukosaepithelien und nachfolgend $\mathrm{zu}$ einer erheblichen Blutung in das Darmlumen, die das Tier nicht überlebte. Die Befunde, die diese Erklärung stützen, sind die großen Blutmengen im Zäkum und im Colon descendens sowie die subendokardialen Blutungen des linken Ventrikels, die bei der Todesursache Verbluten relativ häufig festgestellt werden [19]. 


\section{Fazit für die Praxis \\ - Bei der Klärung der Todesursache von bestimmten Zoo- und Wildtie- ren kann die Rechtsmedizin, ggf. in Kooperation mit weiteren Fach- richtungen der Humanmedizin, die Veterinärmedizin unterstützen. \\ - Ein Orang-Utan in Zoohaltung kann bei einer Vorerkrankung des Darmes akut an einer Escherichia-coli-Enteritis versterben, ohne dass andere, in der unmittelbaren Umgebung lebende Primaten zur gleichen Zeit an einer Enteritis erkranken müssen. \\ - Das Gebiet der forensischen Vete- rinärmedizin beschränkt sich nicht ausschließlich auf Aufträge durch Staatsanwaltschaften.}

\section{Korrespondenzadresse}

Prof. Dr. med. F. Zack

St.-Georg-Straße 108, 18055 Rostock,

Deutschland

fred.zack@med.uni-rostock.de

H. Nizze Emeritus, Institut für Pathologie, Universitätsmedizin Rostock, Rostock, Deutschland

Funding. Open Access funding enabled and organized by Projekt DEAL.

\section{Einhaltung ethischer Richtlinien}

Interessenkonflikt. F. Zack, P. Warnke, J. Manhart, A. Angeli, H. Nizze, J.O. Rudnick, I. Steinhagen, V. Kolbe, M. Hammer und A. Büttner geben an, dass kein Interessenkonflikt besteht.

Für diesen Beitrag wurden von den Autoren keine Studien an Menschen oder Tieren durchgeführt. Für die aufgeführten Studien gelten die jeweils dort angegebenen ethischen Richtlinien. Für Bildmaterial oder anderweitige Angaben innerhalb des Manuskripts, über die Patienten zu identifizieren sind, liegt von innen und/oder ihren gesetzlichen Vertretern eine schriftliche Einwilligung vor.

Open Access. Dieser Artikel wird unter der Creative Commons Namensnennung 4.0 International Lizenz veröffentlicht, welche die Nutzung, Vervielfältigung, Bearbeitung, Verbreitung und Wiedergabe in jeglichem Medium und Format erlaubt, sofern Sie den/die ursprünglichen Autor(en) und die Quelle ordnungsgemäß nennen, einen Link zur Creative Commons Lizenz beifügen und angeben, ob Änderungen vorgenommen wurden.

Die in diesem Artikel enthaltenen Bilder und sonstiges Drittmaterial unterliegen ebenfalls der genannten Creative Commons Lizenz, sofern sich aus der Abbildungslegende nichts anderes ergibt. Sofern das be- treffende Material nicht unter der genannten Creative Commons Lizenz steht und die betreffende Handlung nicht nach gesetzlichen Vorschriften erlaubt ist, ist für die oben aufgeführten Weiterverwendungen des $\mathrm{Ma}$ terials die Einwilligung des jeweiligen Rechteinhabers einzuholen.

Weitere Details zur Lizenz entnehmen Sie bitte der Lizenzinformation auf http://creativecommons.org/ licenses/by/4.0/deed.de.

\section{Literatur}

1. Jonas L, Krone O, Zack F (2005) Blei- und Antimonnachweis in Geschosspartikeln aus Seeadlermägen. In: Krone O, Hofer H (Hrsg) Bleihaltige Geschosse in der Jagd - Todesursache von Seeadlern? Institutfür Zoo- und Wildtierforschung (IZW), Berlin, S18-23

2. Zack F, Wegener R (2002) Tötung eines Wolfes - ein außergewöhnlicher Fall forensischer Veterinärpathologie. Arch Kriminol 209:116-122

3. Banaschak S, Sliwa A (2011) Vaterschaftsbestimmung durch DNS aus Speichelproben bei Bonobos. ZKölner Zoos 54:33

4. Cawthorn Lang KA (2005) Primate factsheets: orangutan (Pongo) taxonomy, morphology, \& ecology. http://pin.primate.wisc.edu//factssheet/ entry/orangutan. Zugegriffen:30. März 2020

5. Kanamori T, Kuze N, Bernhard H, Malim TP, Koshima S (2012) Fatality of a wild Bornean orangutan (Pongo pygmaeus morio): behavior and death of a wounded juvenile in Danum Valley, North Borneo. Primates 53:221-226

6. Mijagi J, Tsuhako K, Kinjo T, Iwamasa T, Kamada Y, Kinju T, Konayagi Y (1999) Coxsackie B4 myocarditis in an orangutan. Vet Pathol 36:452-456

7. Ketz-Riley CJ, Kennedy GA, Carpenter JW, Zeidner NS, Petersen JM (2009) Tularemia type a in captive Bornean orangutans (Pongo pygmaeus pygmaeus). JZoo WildI Med 40:257-262

8. Murray S, Zdziarski JM, Bush M, Citino SB, Schulman FY, Montali R (2000) Diverticulitis with rupture and fatal peritonitis in a Sumatran orangutan (Pongo pygmaeus).Comp Med 50:452-454

9. Ihms EA, Daniels JB, Koivisto CS, Barrie MT, Russel DS (2014) Fatal Streptococcus anginosusassociated pneumonia in a captive Sumatran orangutan (Pongo abelii). J Med Primatol 43:48-51

10. Cambre RC, Wilson HL, Spraker TR, Favara BE (1980) Fatal airsacculitis and pneumonia, with abortion, in an orangutan. J Am Vet Med Assoc 177:822-824

11. Wiesner H, Gedek B (1973) Behandlungserfolge einer chemotherapieresistenten Enteritis beim Orang-Utan (Pongo pygmaeus). In: Ippen R, Schröder HD (Hrsg) Erkrankungen der Zootiere. Verhandlungsbericht des XV. Internationalen Symposiums über die Erkrankungen der Zootiere vom 27. Juni bis 01. Juli in Kolmarden. Akademie, Berlin, S287-291

12. Andreas NJ, Kampmann B, Mehring-Le-Doare K (2016) Human breast milk: a review on its composition and bioactivity. Early Hum Dev 91:629-635

13. Buckle A, Taylor C (2017) Cost and cost-effectivness of donor human milk to prevent necrotizing enterocolitis: systematic review. Breastfeed Med 12:528-536

14. PatelAL, Kim JH(2018) Human milkand necrotizing enterocolitis. Semin Pediatr Surg 27:34-38

15. Mirsepal-Lauridsen HC, Vallance BA, Krogfelt KA Petersen AM (2019) Escherichia coli pathobionts associated with inflammatory bowel disease. Clin Microbiol Rev 32:e00060-18

16. Schuetz AN (2019) Emerging agents of gastroenteritis: Aeromonas, Plesiomonas, and the diarrheagenic pathotypes of Escherichia coli. Semin Diagn Pathol 36:187-192

17. Bielaszewska M, Mellmann M, Zhang W, Köck $R$ Fruth A, Bauwens A, Peters G, Karch H (2011) Characterisation of the Escherichia coli strain associated with an outbreak of haemolytic uraemic syndrome in Germany, 2011: a microbiological study. Lancet Infect Dis 11:671-676

18. LohnerL,SperhakeJP,PüschelK,EdlerC(2020)Zwei plötzliche Todesfälle bei chronischer Obstipation. Rechtsmedizin 30:38-43

19. Plattner T, Yen K, Zollinger U (2008) The value of subendocardial haemorrhages as an indicator of exsanguinationj and brain injury-a retrospective forensic autopsy study. J Forensic Leg Med 15:325-328 